\title{
Search for the neutral MSSM Higgs bosons with ATLAS
}

\author{
Jana Schaarschmidt on behalf of the ATLAS Collaboration
}

Institutf. Kern- und Teilchenphysik, 01069 Dresden, Germany

\begin{abstract}
The discovery of a neutral Higgs boson with large branching fractions into tau or muon pair final states would be a strong evidence of New Physics beyond the Standard Model. The discovery potential of these processes with the ATLAS detector at the Large Hadron Collider for integrated luminosities of $10 \mathrm{fb}^{-1}$ and $30 \mathrm{fb}^{-1}$ is presented. The studies are based on the analysis of fully simulated Monte Carlo samples.
\end{abstract}

Keywords: SUSY08, MSSM Higgs, LHC, ATLAS

PACS: $12.60 . J v, 14.80 . \mathrm{Cp}$

\section{INTRODUCTION}

The discovery of the Higgs bosons and of signatures beyond the Standard Model is the main objective of ATLAS.

In the Minimal Supersymmetric Extension of the Standard Model (MSSM) five Higgs bosons are expected, three of them are neutral $(h / H / A)$ and two of them are charged $\left(H^{ \pm}\right)$. At tree level their properties are determined by two parameters: the mass of the $\mathrm{CP}$ odd boson $A$ and the ratio of the expectation values, $\tan \beta$, of the two Higgs doublets. In the MSSM the coupling of the Higgs boson to down-type fermions is enhanced, and channels which have limited sensitivity in the SM become most important.

The MSSM Higgs bosons are either produced via the gluon fusion process $(g g \rightarrow A / H / h)$ which dominates for low $\tan \beta$ values, or via $b$ quark associate production. In the latter case a $b$-tagging requirement can be used to suppress background processes.

The mass differences and the widths of the Higgs bosons are a function of $\tan \beta$. For some regions of the parameter space $\left(m_{A} \sim 130 \mathrm{GeV}\right.$ and low $\left.\tan \beta\right)$ this mass difference and the intrinsic width are of comparable size. This offers the potential to observe all three neutral Higgs bosons in the $\mu^{+} \mu^{-}$final state thanks to the excellent dimuon mass resolution of the ATLAS detector.

This paper describes the search for neutral MSSM Higgs bosons in their decays to $\mu$ or $\tau_{\ell}$ pairs in the mass range from $110 \mathrm{GeV}$ up to $450 \mathrm{GeV}$ in the $m_{h}^{\max }$ benchmark scenario [1]. In these studies (N)NLO cross sections have been used whenever available.

A detailed description of the ATLAS detector and its performance is given in [2]. Details of the Monte Carlo data as well as details of the analyses are given in [3].
SEARCH FOR A $/ \mathbf{H} / \mathbf{h} \rightarrow \mu \mu$

\section{Event Selection}

The experimental signature of this final state is two isolated muons of opposite charge and no missing transverse energy $\left(\mathbb{E}_{T}\right)$. The events are triggered by a single high $p_{T}$ muon trigger. The muons are required to have $p_{T}>20 \mathrm{GeV}$ and a pseudorapidity $|\eta|<2.7$. The reconstructed di-muon mass has an excellent resolution $\sigma$ of a few $\mathrm{GeV}$ depending on $m_{A}$.

Relevant backgrounds in this channel are $Z$ boson production (with subsequent $Z$ decay into two muons) and top-pairs. A cut of $\mathbb{E}_{T}<40 \mathrm{GeV}$ has been applied to reduce $t \bar{t}$ which has higher missing energy due to the presence of neutrinos. The $Z$ background can be suppressed by requiring one or more $b$-jets. The $t \bar{t}$ background is suppressed by requiring a $b$-veto. The analysis has therefore been split to follow both strategies.

The major backgrounds in the $b$-tagged case are that from $Z+b$ jets, mistagged $Z+$ light jets and from $t \bar{t}$ events, the latter being dominant. Therefore, further cuts on the dimuon azimuthal angle and on the scalar sum of the $p_{T}$ of all jets are imposed to reduce it. The irreducible $Z+$ jets background is dominant in the $b$-vetoed analysis. Finally - for both analyses - a mass window cut $\Delta m=m_{A} \pm 2 \sigma$ has been applied. The invariant dimuon mass after selection for both analysis approaches are shown in Fig. 1.

\section{Background Estimation}

Since the normalization of the background has a large theoretical uncertainty coming from the choice of the parton-density functions and that of the renormalization and factorization scale, methods to estimate the background directly from data have been developed. The side 

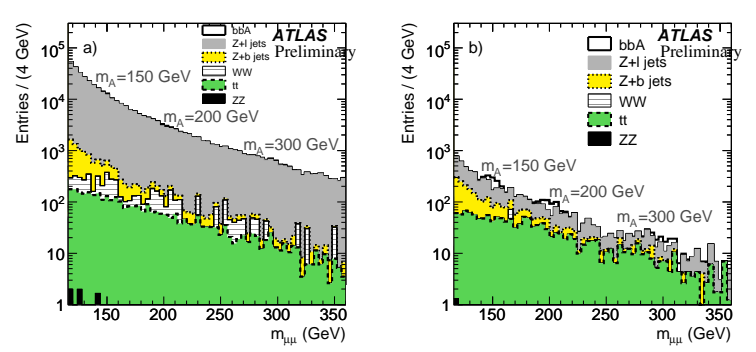

FIGURE 1. Resulting di-muon mass spectra for the $b$-vetoed (left) and $b$-tagged analysis (right). In the $b$-vetoed analysis the $Z+$ jets background is dominating. Both gluon fusion and $b \bar{b} A$ processes contribute to the signal. In the $b$-tagged case only $b \bar{b} A$ contributes to the signal and $t \bar{t}$ and $Z+j e t s$ are equally important backgrounds.

bands of the dimuon mass distribution together with control samples are used for this purpose. A fit to the dimuon mass distribution is performed using a Breit-Wigner plus an exponential term to describe the background and a Gaussian shape for the signal. The shape of the background can be controlled selecting a $Z \rightarrow e^{+} e^{-}$sample using the same selection criteria described above since the contribution from signal events is negligible in that case.

Further handles to control the $t \bar{t}$ background are provided by using events with an $e-\mu$ final state and/or by inverting the $t \bar{t}$ oriented cuts ( $E_{T}^{\text {miss }}$ and $\sum p_{T}$ of the jets). Both cases result in a fairly clean $t \bar{t}$ sample with the same shape distribution as the background under the signal.

That way an uncertainty of $\sim 10 \% / \sqrt{\mathscr{L}\left[\mathrm{fb}^{-1}\right]}$ for the $b$-tagged analysis and $\sim 2 \% / \sqrt{\mathscr{L}\left[\mathrm{fb}^{-1}\right]}$ for the $b$-vetoed analysis are expected.

\section{Discovery Potential}

The signal significance is evaluated using the profile likelihood method [4]. The experimental uncertainties include the reconstruction efficiency, momentum resolution and momentum scale for the muons, the jet energy scale and resolution as well as the uncertainty on the $b$ tagging efficiency and the light-jet rejection rate. Figures 2 and 3 show the discovery and exclusion contours for the combination of the $b$-vetoed and $b$-tagged analyses.

The $\mu \mu$ channel offers the prospect of Higgs mass measurement and exclusion power in addition to the $\tau \tau$ final state.

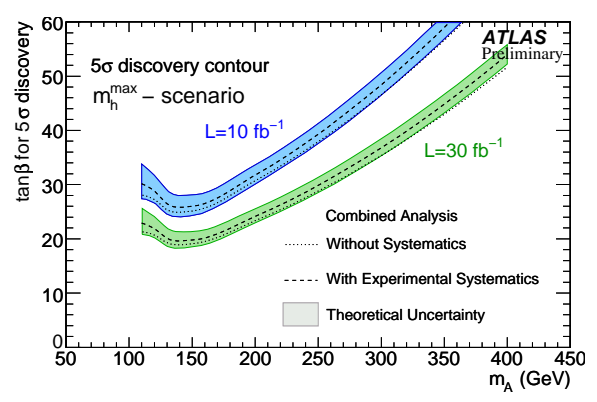

FIGURE 2. Discovery contour for the combination of $b$ tagged and $b$-vetoed analysis for $10 \mathrm{fb}^{-1}$ and $30 \mathrm{fb}^{-1}$ as indicated in the plot. The dashed (dotted) lines indicate the discovery contours with (without) systematic uncertainties taken into account. The bands show the theoretical uncertainty.

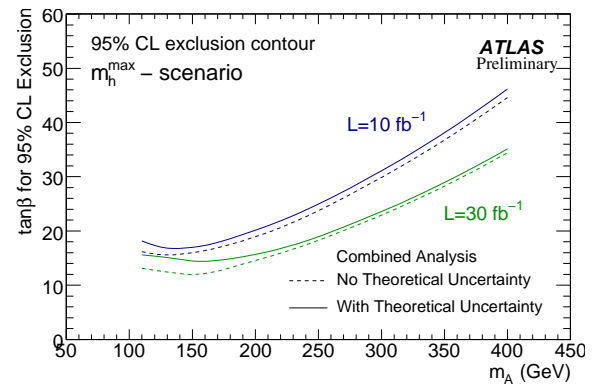

FIGURE 3. $95 \% \mathrm{CL}$. exclusion contour for the combination of $b$-tagged and $b$-vetoed analysis for $10 \mathrm{fb}^{-1}$ and $30 \mathrm{fb}^{-1}$ as indicated in the plot. The dashed (dotted) lines indicate the discovery contours with (without) systematic uncertainties taken into account. The dashed lines do not include the theoretical uncertainties.

$$
\text { SEARCH FOR A } / \mathbf{H} / \mathbf{h} \rightarrow \tau \tau \rightarrow 2 \ell+\mathbf{4} v
$$

\section{Event Selection}

The signal signature in this channel consists of two oppositely charged leptons with $p_{T}>6 \mathrm{GeV}$ and $|\eta|<$ 2.5 , and missing transverse energy due to the presence of neutrinos in the final state. The events are triggered by a high $p_{T}$ single- or di-lepton trigger. The Higgs boson mass can be fully reconstructed using the collinear approximation [5]. This leads to an $m_{A}$ dependent mass resolution about one order of magnitude larger than the intrinsic width.

Relevant backgrounds are $Z \rightarrow \tau \tau+$ jets, $Z \rightarrow \ell \ell+$ jets $W \rightarrow \ell v+$ jets and $t \bar{t}$. At least one $b$-jet is required to suppress light jet contributions, hence only $b \bar{b} A$ contributes to the signal. Events with more than two jets are rejected to suppress top-pairs. In the di-lepton final state with its clean signature the QCD background is supposed to be negligible.

A Higgs boson mass dependent selection based on 
kinematic variables has been optimized for the best statistical significance. In order to suppress $t \bar{t}$ cuts on the $p_{T}$ of the leading $b$-jet and $\mathscr{E}_{T}$ are applied. The latter cut and a cut on $m_{\ell \ell}$ strongly reduces $Z \rightarrow \ell \ell$ events $(\ell \in e, \mu)$. A cut on $\Delta \Phi_{\ell \ell}$ is highly Higgs boson mass dependent and reduces top-pairs and $Z \rightarrow \tau \tau$.

The invariant $\tau \tau$ mass distributions for four mass points are shown in Fig. 4. While for low Higgs boson masses the $Z \rightarrow \tau \tau$ background is dominating, the contributions from $t \bar{t}$ become dominant for higher $A$ masses. Contributions from $\mathrm{W}+$ jets and $Z \rightarrow \ell \ell$ are small.
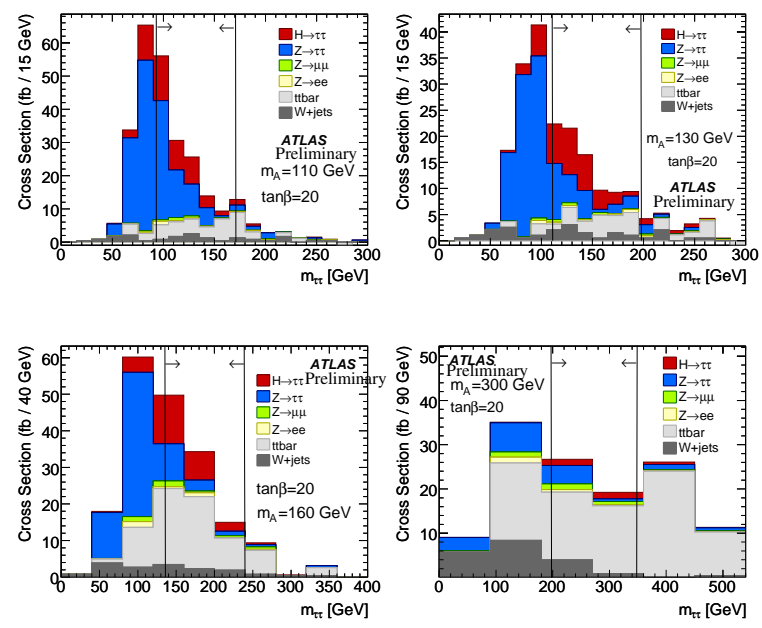

FIGURE 4. Invariant $m_{\tau \tau}$ distribution for Higgs boson masses as indicated in the plots for $30 \mathrm{fb}^{-1}$ and $\tan \beta=20$. In the low $m_{A}$ region the $Z \rightarrow \tau \tau$ background is dominant, for higher values of $m_{A}$ the $t \bar{t}$ background becomes important and is dominant for $m_{A}>200 \mathrm{GeV}$.

The experimental systematic uncertainty on the remaining backgrounds are $4-7 \%$ on $t \bar{t}$ and $\sim 5 \%$ on $\mathrm{W}+$ jets. The irreducible $Z \rightarrow \tau \tau$ background is controlled with an uncertainty of $2.6 \%$ using control samples from data.

\section{Background Estimation}

Control samples containing $Z \rightarrow \ell \ell$ decays with large statistics and high purity can be defined selecting events with $m_{\ell \ell}$ around the $Z$ mass and with less than three jets.

The momentum components and the energy of the muons from $Z \rightarrow \mu \mu$ decays are substituted according to $Z \rightarrow \tau \tau$ reference histograms to obtain the $m_{\tau \tau \rightarrow \mu \mu}$ shape. The $m_{\tau \tau}$ shapes of the other leptonic final states are identical with the $m_{\tau \tau \rightarrow \mu \mu}$ shape within statistical uncertainties. The $Z \rightarrow \tau \tau$ sample obtained with this procedure is then normalized to the $Z \rightarrow \ell \ell$ data, rescaled by the Monte Carlo ratio of the cross sections of the selected $Z \rightarrow \tau \tau \rightarrow \ell \ell$ and $Z \rightarrow \ell \ell$ events in the signal region and the control region. That way systematic uncer- tainties cancel out to a large extent. More details can be found in [3].

Methods to estimate the normalization of the $t \bar{t}$ background from data are under development. In a conservative approach an additional $10 \%$ theoretical uncertainty on the top cross section has been assumed.

\section{Discovery Potential}

The statistical significance is calculated via $S / \sqrt{B+(\Delta B)^{2}}$, where $(\Delta B)^{2}$ includes all contributions from systematic uncertainties. The discovery and exclusion contours for $30 \mathrm{fb}^{-1}$ are shown in Fig. 5 .
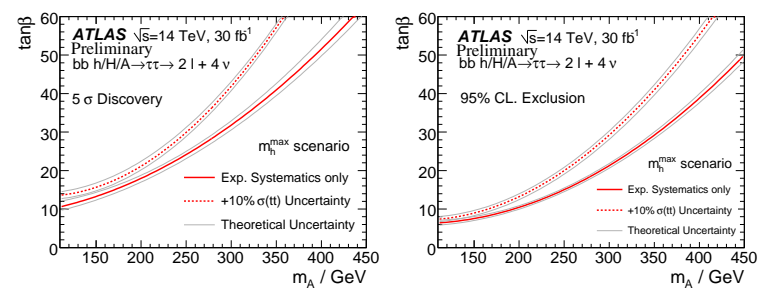

FIGURE 5. $5 \sigma$ discovery contours (left) and $95 \%$ CL. exclusion contours (right) in the $\tau \tau$ channel. The grey lines indicate the theoretical uncertainty on the signal. The solid line shows the contour with experimental systematics only, the dashed lines includes an additional theoretical uncertainty on the $t \bar{t}$ cross section of $10 \%$.

The lepton-lepton channel offers a high discovery potential over large parts of the $m_{A}-\tan \beta$ plane. Studies of the lepton-hadron and hadron-hadron final states are ongoing. They will increase the discovery potential and extend the covered mass range.

\section{ACKNOWLEDGMENTS}

The author thanks all ATLAS collaborators, especially M. Kobel, W. Mader, M. Warsinsky, O. Silbert, E. Gross, S. Horvat, D. Fassouliotis.

\section{REFERENCES}

1. M. S. Carena, S. Heinemeyer, C. E. M. Wagner and G. Weiglein, Eur. Phys. J. 26, 601 (2003).

2. ATLAS Collaboration, JINST $3 \mathbf{S 0 8 0 0 3}$ (2008).

3. ATLAS Collaboration, Expected Performance of the ATLAS Experiment, Detector, Trigger and Physics, CERN-OPEN-2008-020, Geneva, 2008, to appear.

4. J. C. W.A. Rolke, A.M. Lopez, Nucl.Instrum.Meth.A 551, 493-503 (2005).

5. R. K. Ellis, I. Hinchliffe, M. Soldate and J. J. van der Bij, Nucl. Phys. B 297, 221 (1988).

September 12, 2008 